\title{
Analysis of Package Inserts of Drugs Utilized in a Tertiary Care Hospital
}

\author{
Dass Ashwitha Shruti, Narayana Sarala, Krishnaswamy Bhuvana \\ Department of Pharmacology, Sri Devaraj Urs Medical College, Sri Devaraj Urs Academy of Higher Education and Research, Tamaka, Kolar-563101, Karnataka, INDIA.
}

\begin{abstract}
Aim: To evaluate the Package inserts (PIs) for presentation, accuracy, completeness of clinical information and grade them according to the scores obtained. Material and Methods: An observational study conducted by the Department of Pharmacology, Sri Devaraj Urs Medical College from March to August 2014. Pls were analyzed for five leaflet characteristics and for 25 criteria listed by the Indian Drug and Cosmetic Rules, 1945 Each criteria was scored for presence of information as ' 1 ', absence as ' 0 '. Total score of $>20$ was graded as ' $A$ ', $10-20$ as ' $B$ ' and $<10$ as ' $C$ '. Data was expressed as percentage. Results: Three hundred and sixty three Pls were analyzed. Considering the quality and texture, $80.2 \%$ were categorized as good and $19.8 \%$ as bad. All Pls were legible. Generic names were used in $99.2 \%$. Content of active ingredients, indication for use were listed in $96.9 \%$ and $99.2 \%$ respectively. Posology details were mentioned in $97.2 \%$ and contraindications were given in $91.5 \%$ Pls. Undesirable effects were listed in 92.6\%. Manufacturer's name and address was provided in $88.1 \%$. PI with grade A, B and C were $10.2 \%, 83.8 \%$ and $6.1 \%$ respectively. Conclusion: Majority of the Pls were of grade B which suggests that most of the relevant information was mentioned except few criteria.
\end{abstract}

Key words: Information tool, Package insert, Rational prescribing,

Posology, Generic name.

\section{PICTORIAL ABSTRACT}

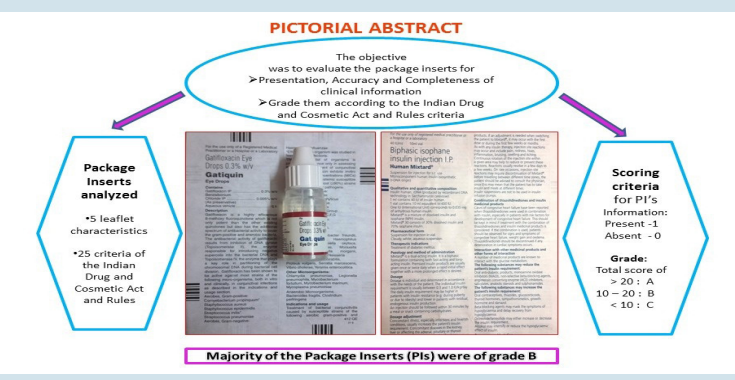

Correspondence :

Dr. Sarala Narayana,

Professor and Head, Department of Pharmacology, Sri Devaraj Urs Medical College, Sri Devaraj Urs Academy of Higher Education and Research, Tamaka Kolar- 563101, Karnataka, INDIA.

Phone no: 09845750165

E-mail: n_sarala@rediffmail.com

DOI: 10.5530/jyp.2016.3.18

\section{INTRODUCTION}

Package insert (PI) is a document provided along with drug, it is also known as prescription drug label or prescribing information. ${ }^{1}$ This provides the patient complete information in terms of the purpose, benefits and risks of the drug prescribed. ${ }^{2}$ The PI is thus considered an important source of information for patients and health care providers in developing countries because of limited access to recent information about drugs. ${ }^{3}$

The prescribing physician plays an important role in providing drug information to most of the patients. An effective communication between the physician and the patients may not always be practically possible due to an inadequate doctor-patient ratio in India. A study has reported that low level of health literacy among patients and physician's inability to convey complete information about the drug, as barriers for patientphysician communication. ${ }^{4}$ Oral instructions are likely to be missed, misunderstood or forgotten. Hence, it is important that patients to certain extent require written information in order to use the drugs judicially. ${ }^{5}$ In India, the concept of package insert is governed by the 'Drugs and Cosmetics Act (1940) and Rules (1945). The section 6 of Schedule D (II) of the rules lists the headings according to which information should be provided in the package inserts. ${ }^{6}$ A good PI should contain complete, approved, essential and accurate information about a drug. ${ }^{1}$

Incomplete and incorrect product information may result in irrational prescribing leading to undesirable effects. Hence, this information tool must be constantly updated as and when relevant preclinical and clinical data is available. ${ }^{5}$ Therefore this study was carried out to critically assess the PIs using the criteria laid down by Indian Drug and Cosmetic Rules,
1945. PI were evaluated for presentation, accuracy, completeness of clinical information and they were graded based on their scores.

\section{MATERIALS AND METHODS}

An observational study was conducted by the Department of Pharmacology, Sri Devaraj Urs Medical College from March to August 2014. The protocol was approved by Institutional Ethics Committee. The study was carried out by collecting 363 package inserts from the pharmacy and wards of RL Jalappa Hospital and Research Centre.

All the package inserts were analyzed for five leaflet characteristics: Dimensions and layout, type of paper, absence of transparency, colour of paper and text, use of headings, pictograms or graphics. The PIs were also evaluated for the 25 parameters based on criteria laid down by Indian Drug and Cosmetic Rules, 1945: Legibility, approved generic name of active ingredients, content of active ingredient per dosage form, generic names of other ingredients, therapeutic indications, posology and method of administration, contraindications, special warnings and precautions, drug interactions, pregnancy and lactation, pediatric and geriatric indications, special conditions and contraindications, effect on ability to drive and use machines, undesirable effects, drug dose, over dosage, pharmacokinetic information, storage information, instructions for use and handling, shelf life, date on which information was last updated, name and address of manufacturer/distributor, provision of full information on request should be highlighted, retail price of the drug and references. Each criteria was scored for presence of information as ' 1 ', absence as ' 0 '. Total score of $>20$ was graded as 'A', $10-20$ as 'B' and $<10$ as 'C'. Descriptive statistics was used to analyze the data and expressed as percentage. 


\section{RESULTS}

Three hundred and sixty three package inserts were analyzed. Considering the leaflet characteristics based on quality and texture, $80.2 \%$ $(n=291)$ were categorized as good and $19.8 \%(n=72)$ as bad. Pictograms and graphics were present in $9.4 \%$ of PIs for better explanation of instructions. Average length and breadth were $22.35 \pm 11.32 \mathrm{~cm}$ and $14.7 \pm$ $7.49 \mathrm{~cm}$ respectively. White colour background and black text was used in $75.2 \%$. The extent to which PIs followed the 25 criteria laid down by the Drug and Cosmetic Rules, 1945 is represented in Table 1.

Grade A suggests the PIs were good, B average and C poor category majority of them were graded B (Figure 1). Thirty seven PIs belonged to grade A, Ranbaxy laboratories limited had three PIs with grade A of which two had score of 24 and one had 23 (Figure 2). The PIs were categorized based on the scores obtained into grade $\mathrm{B}$ and $\mathrm{C}$ and maximum belonged to grade B (Figure 3). Package inserts were present in most of the cartons of tablets and injections (Figure 4) and highest was PIs with antimicrobials (Figure 5).

\section{DISCUSSION}

Package inserts belonging to different countries are approved by their regulatory bodies. Food and Drug Administration is the regulatory authority for United States, European Medicines Agency for Europe whereas in India it is the Ministry of Health and Family Welfare. Full prescribing information is submitted by the pharmaceutical companies as a part of the new drug application for marketing to the regulatory authorities. ${ }^{7}$ Once the application is approved, the information is accompanied with the drug in the drug cartons.

In India, the regulations for manufacture, distribution and sale of pharmaceutical products are present in Section 6 of Schedule D (II) of the Rules which relates to labelling, listing the headings according to which information should be provided in the package inserts. The headings

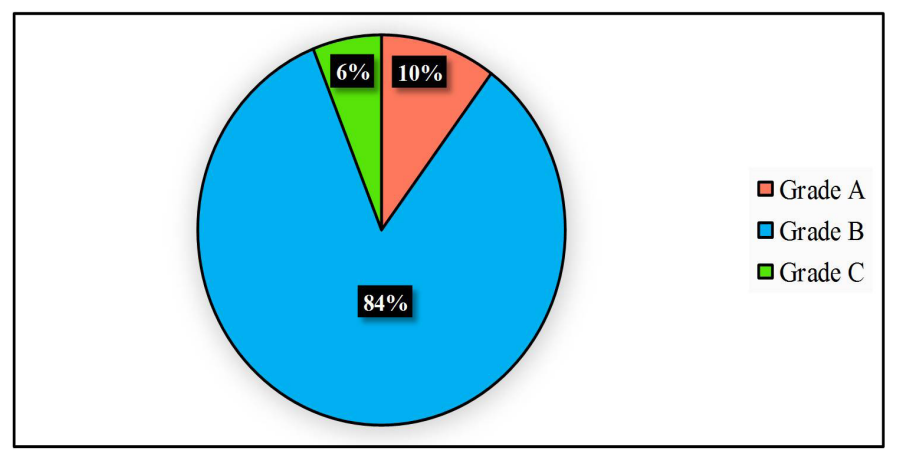

Figure 1: Grades of package inserts.

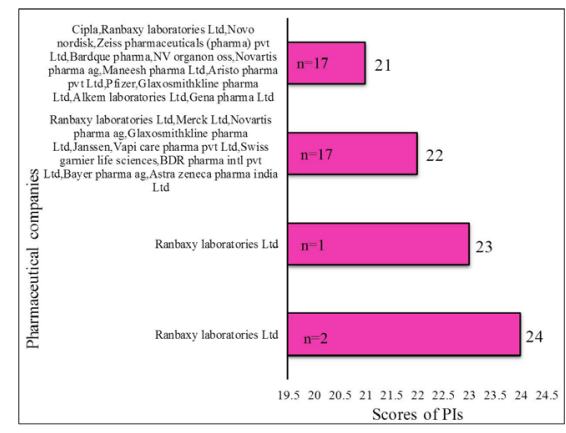

Figure 2: Package inserts that scored 21-25 (Grade A).

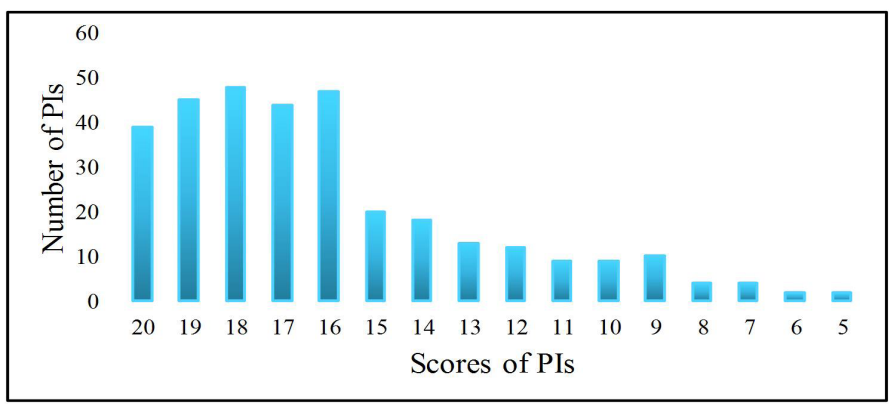

Figure 3: Package inserts categorized based on scores (Grade B and C).

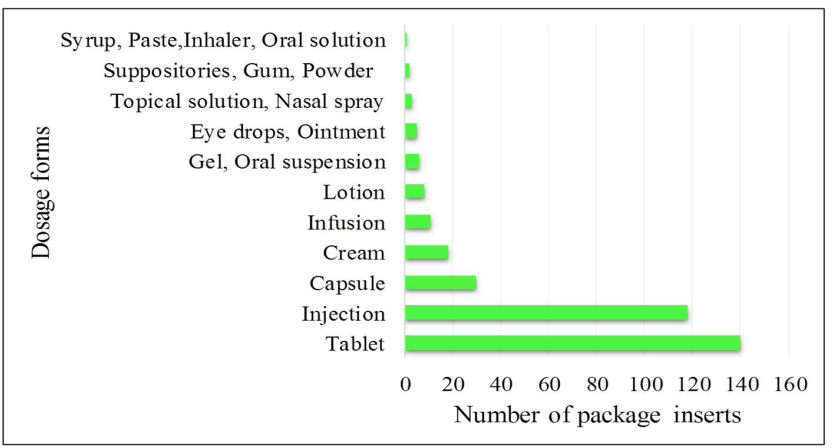

Figure 4: Package inserts based on the dosage forms.

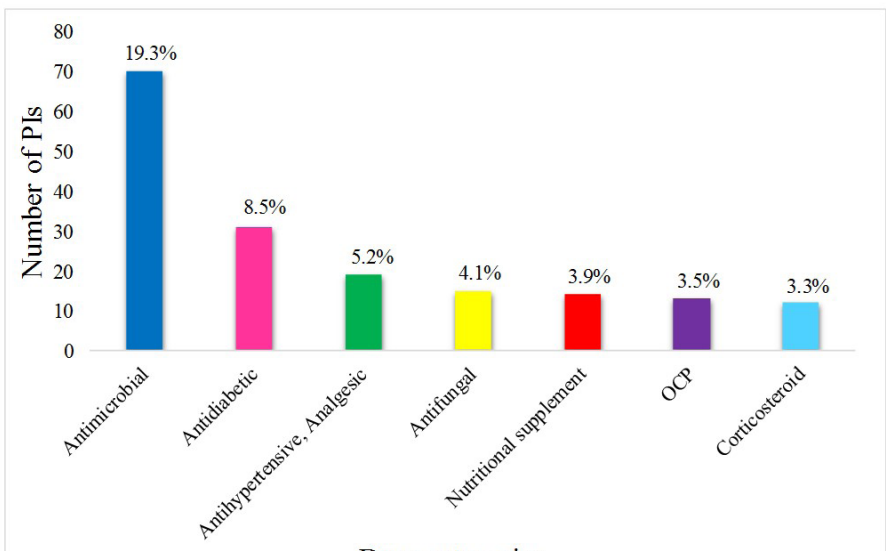

Drug categories

Figure 5: Categories of package inserts.

are grouped under two broad sections, Section 6.2 and 6.3 pertaining to importance of clinical use of the drug and pharmaceutical information respectively. ${ }^{6}$ We summed and included all the headings as 25 criteria for which the PIs were analyzed.

Leaflet characteristics of PI are important because no matter how good the information is, the paper should be presentable and have uniform characteristics. In our study, there was no uniformity in length and breadth and it was clumsy to handle an over-sized leaflet. There was no uniformity in font size which made it difficult for analyzing. The lack of uniformity in size, shape and font causes inconvenience not only to the prescribers but the patients as well. In a study regarding leaflet characteristics, it was reported that most of them were not satisfactory and the content in terms of readability, understanding should have been improved. ${ }^{2}$ 
Table 1: Package inserts that followed criteria laid down by Drug and Cosmetic Rules, 1945

\begin{tabular}{|c|c|c|}
\hline Criteria & Mentioned (\%) & Not mentioned(\%) \\
\hline Legibility (Yes/ No) & 100 & 0 \\
\hline Approved generic name of active ingredients & 99.2 & 0.8 \\
\hline Therapeutic indications & 99.2 & 0.8 \\
\hline Posology and method of administration & 97.2 & 2.8 \\
\hline Content of active ingredient per dosage form & 97 & 3 \\
\hline Special warnings and precautions & 93.4 & 6.6 \\
\hline Undesirable effects & 92.6 & 7.4 \\
\hline Contraindications & 91.5 & 8.5 \\
\hline Drug dose & 89.3 & 10.7 \\
\hline Storage information & 88.1 & 11.9 \\
\hline Name and address of manufacturer/distributor & 88.1 & 11.9 \\
\hline Drug interactions & 80.4 & 19.6 \\
\hline Pregnancy and lactation & 79.1 & 20.9 \\
\hline Pharmacokinetic information & 77.7 & 22.3 \\
\hline Over dosage & 74.4 & 25.6 \\
\hline Pediatric and geriatric indications & 65.8 & 34.2 \\
\hline Generic names of other ingredients & 62.3 & 37.7 \\
\hline Instructions for use and handling & 51.8 & 48.2 \\
\hline Special conditions and contraindications & 32.5 & 67.5 \\
\hline Provision of full information on request-highlighted & 30.9 & 69.1 \\
\hline Shelf life & 24.5 & 75.5 \\
\hline Date on which information was last updated & 18.7 & 81.3 \\
\hline Effect on ability to drive and use machines & 17.6 & 82.4 \\
\hline References & 9.4 & 90.6 \\
\hline Retail price of the drug & 0 & 100 \\
\hline
\end{tabular}

The quality and texture was good in $80.2 \%$. The quality was assessed based on absence of transparency of the paper i.e. when the paper was placed on separate sheet with written text, a good quality paper would not reveal the text but bad would reveal it. The texture was assessed whether the written text on the reverse side of sheet is seen through while reading. Less than $10 \%$ had used pictograms and graphics. Pictorial representation should be increased since it adds value to the better understanding of the instructions from patient's point of view, especially from the rural areas. ${ }^{8}$ Information on PIs can also be made available in local languages as India is a country with many languages and most people are not familiar with English. In our study, only three PIs had information written in many languages but the disadvantage was that it had occupied a lot of space. All PIs used headlines to provide information, majority used white background and with black text.

Fuchs et al study did a survey on patients where majority $(79.6 \%$ of all volunteers) said that they "always" read the PIs, $19.3 \%$ read "sometimes" and only $1.1 \%$ "never" read them. ${ }^{9}$ Gupta VK et al study also had similar result where majority i.e. $58 \%$ of all medical students read the PIs. ${ }^{3}$ This indicates the need for patient oriented PIs which provide the necessary information needed by common man. Fuchs et al study also ranked the criteria in the order of importance which is therapeutic indications, dosage instruction, ingredients, appropriate precautions for use, special warnings, contraindications, interactions, possible adverse drug reactions, therapeutic group, hints for application errors, application form and quantity of the drugs, storage, manufacturer, date of the last update. ${ }^{9}$ In our study, most ( $80 \%)$ of the PIs fulfilled the above criteria but only
$18.7 \%$ mentioned the date of last update (Table 1). Mentioning this is important so that the patient's and physician's trust will be increased for the drug prescribed.

In addition, we have also analyzed the generic names of other ingredients, use in pregnancy and lactation, pediatric and geriatric indications, pharmacokinetic information, provision of full information on request, shelf life, date on which information was last updated, effect on ability to drive and use machines, references and retail price of the drug (Table 1). The last five criteria were mentioned in less than $25 \%$ of PIs. Details about effects of the drugs in the special conditions and driving are needed to avoid harm to patients. Information on shelf life is important as the drug that has passed its shelf life may still be safe for consumption but its quality cannot be assured and can lead to poor control of diseases like diabetes mellitus and hypertension. It is also important to list the inactive ingredients, such as sodium salts or artificial sweeteners because patients may be allergic to them. Contact details in case of any query should always be available. Retail prices have not been mentioned in any of the PIs, it would be beneficial for the patient if it is quoted.

We have observed that PIs were not provided with all the medications, it should be made mandatory. The PI can have an impact on the usefulness of drug usage by patients as it is seen only after receiving the medication. ${ }^{10}$ PIs are currently viewed as a legal formality rather than an effective tool for guidance. ${ }^{11}$ Providing accurate and complete information about precautions, adverse effects, will promote rational use of medications. 
There should be stringent rules to ensure that the pharmaceutical companies follow the regulatory guidelines regarding the structure, format of the PI so that there are no discrepancies in the 25 criteria. Therefore the requirement presently is to refine the contents of the PIs to make them complete, reliable and up to date. This can be a step forward for ethical and effective distribution of healthcare services in our society.

\section{CONCLUSION}

Majority of the PIs were of grade B which suggests that most of the criteria of the Drugs and Cosmetic act were met but the ultimate aim of all pharmaceutical companies should be to have Grade A quality package inserts. The PI should be made mandatory and strict guidelines need to be implemented for uniformity of leaflet characteristics. Fulfilment of 25 criteria needs to be encouraged for propagation of knowledge and patient care.

\section{ACKNOWLEDGEMENT}

None.

\section{CONFLICT OF INTEREST}

The author declare no conflict of interest.

\section{ABBREVIATIONS USED}

PI: Package Insert.

\section{ABOUT AUTHORS}

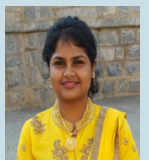

Dr. Ashwitha Shruti Dass: Is a final year post graduate student in MD Pharmacology at Sri Devaraj Urs Medical College, Sri Devaraj Urs Academy of Higher Education and Research, Karnataka, India.

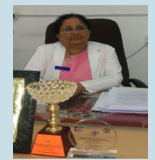

Dr Sarala.N: Is Professor \& HOD Pharmacology and also Director University Department of Medical Education at Sri Devaraj Urs Medical College, SDAUHER, Kolar. Trained in Clinical Pharmacology at Seth G S Medical College,Mumbai and National Course on Educational Science for Teachers of Health Professionals from JIPMER Pondicherry. Is a FAIMER fellow from PSG Coimbatore. Co-investigator for In Hospital Prospective stroke Registry (INSPIRE) study by NIH,USA and Principal Investigator for Situational analysis of the practice patterns in cardiovascular disease management in all Regions of India using mixed research methods (SPECTRUM). Presented papers at national and international conferences and has more than 40 scientific publications to her credit. Peer Reviewer for Journals and Member of various Boards. Coordinator of Pharmacovigilance centre. Organizes workshops on faculty development programs and orientation courses.

\section{REFERENCES}

1. Ramdas D, Chakraborty A, Swaroop HS, Faizan S, Kumar PV, Srinivas BN A study of package inserts in Southern India. J Clin Diagnos Res. 2013;7(11):2475-7.

2. Al-aqeel SA. Evaluation of medication package inserts in Saudi Arabia. Drug Health Patient Saf. 2012:4:33-8.

3. Gupta VK, Pathak SS. Assessment of awareness and knowledge about package inserts amongst medical students: a questionnaire based study. IOSR J Pharm. 2012;2(2):215-7.

4. Khan TM, Hassali MA, Al-Haddad MSM. Patient-physician communication barrier A pilot study evaluating patient experiences. J Young Pharm. 2011;3(3):250-5.

5. Mahatme MS, Dakhale GN, Hiware SK, Wankhede SS, Salve AM, Mahatme SR. Comparison of Indian package inserts in public and private sector: an urgent need for self regulation. Int J Basic Clin Pharmacol. 2013:2(2):165-9.

6. The Drugs and Cosmetics Act and Rules: Ministry of Health and Family Welfare Government of India 2003.p 312. Available from www.emergogroup.com/sites/ default/files/file/india-drugs-and-cosmetics-act-no-23.pdf [Last accessed on 02/04/2016

7. Sawalha AF, Sweileh WM, Zyoud SH, Jabi SW. Comparative analysis of patient package inserts of local and imported anti-infective agents in Palestine. Libyan J Med. 2008;3(4):181-5

8. Rahim N, Rafiq K. Judgement of pharmaceutical package insert available in Pakistan from local and multinational companies. Asian J Pharm Hea Sci. 2013;3(1):597-600.

9. Fuchs J, Hippius M, Schaefer M. A survey of package inserts use by patients. Hosp pharm Europe. 2005;21:29-31.

10. Thorton RG. Package inserts and the standard of care. Proc (BaylUniv Med Cent) 2003;16(4):502-4.

11. Shivkar YM. Clinical information in drug package inserts in India. J Postgrad Med. 2009;55(2):104-7. 